\title{
Interações entre Monensina Sódica, Óleo de Soja e Fontes de Nitrogênio no Desempenho de Novilhos Aberdeen Angus em Confinamento
}

\author{
Rogério de Paula Lana1, Danny G. Fox ${ }^{2}$
}

\begin{abstract}
RESUMO - Quarenta novilhos Aberdeen Angus foram usados para avaliar o efeito da monensina, óleo de soja e fontes de nitrogênio (farelo de soja ou uréia) sobre o desempenho de novilhos em dietas com 90\% de concentrado. A monensina diminuiu o consumo de matéria seca em dietas contendo farelo de soja e o peso corporal aos 56 e 112 dias. O óleo diminuiu o ganho de peso nos períodos de 57-112 dias e 1-112 dias, enquanto a monensina diminuiu o ganho de peso nestes períodos nas dietas contendo farelo de soja como fonte de nitrogênio. O óleo de soja piorou a eficiência alimentar em todos os períodos e o farelo de soja, comparado com dietas com uréia, nos períodos de 57-112 dias e 1-112 dias. A monensina melhorou a eficiência alimentar em 7,4\% para as dietas contendo farelo de soja, na ausência de óleo de soja, mas não alterou a eficiência alimentar para as dietas contendo uréia. Por outro lado, a monensina piorou a eficiência alimentar em $13 \%$ para as dietas contendo farelo de soja e óleo de soja.
\end{abstract}

Palavras-chave: confinamento, eficiência alimentar, monensina, novilhos, óleo, proteína

\section{Interactions Among Monensin, Soybean Oil and Nitrogen Source on Feedlot Performance of Aberdeen Angus Steers}

\begin{abstract}
Fourty Aberdeen Angus steers were used to evaluate the effects of monensin, soybean oil and nitrogen source (soybean meal or urea) on feedlot performance of steers fed $90 \%$ concentrate diets. The monensin decreased dry matter intake of soybean meal supplemented diets and decreased the body weight at 56 and 112 days. The soybean oil decreased the average daily gain from days 57 to 112 and 1 to 112 . While, monensin showed a decreased on the average daily gain in these periods in the diets containing soybean meal as nitrogen source. The soybean oil decreased feed efficiency on all periods and soybean meal, as compared to urea diets, in the days from 57 to 112 and 1 to 112 periods. The monensin increased feed efficiency in $7.4 \%$ for the diets containing soybean meal, without soybean oil, but it did not change the feed efficiency for diets containing urea. In contrast, monensin decreased feed efficiency in $13 \%$ for the diets containing soybean meal and soybean oil.
\end{abstract}

Key Words: feedlot, feed efficiency, monensin, oil, protein, steers

\section{Introdução}

Os benefícios da monensina na melhora da conversão alimentar de bovinos em confinamento têm sido atribuídos ao aumento na digestibilidade dos alimentos, às mudanças na relação acetato:propionato e à redução na produção de metano (GOODRICH et al., 1984; RUSSELL e STROBEL, 1989; RAUN, 1992). Estes efeitos, entretanto, explicam de 33 a $50 \%$ da melhora na conversão alimentar (THORNTON e OWENS, 1981; WEDEGAERTNER e JOHNSON, 1983). Outras pesquisas indicaram que a monensina reduz a perda de proteína pela fermentação ruminal em animais recebendo dietas de forragem (DINIUS et al., 1976; YANG e RUSSELL, 1993; LANA e RUSSELL, 1997), sendo este efeito consistente com a habilidade da monensina em inibir as bactérias gram-positivas utilizadoras de amino ácidos como fonte exclusiva de energia e nitrogênio (KRAUSE e RUSSELL, 1996). Trabalhos com bovinos em confinamento, por sua vez, têm demonstrado que a monensina melhora a conversão alimentar, quando fontes de proteína verdadeira são utilizadas em vez de nitrogênio não protéico (HANSON e KLOPFENSTEIN, 1979; LANA et al., 1997).

Lipídio dietético inibe bactérias ruminais grampositivas, aumenta propionato, diminui a produção de metano (RICHARDSON et al., 1976; CHALUPA et al., 1984) e eleva o fluxo de amino ácidos para o intestino delgado (ZINN e PLASCENCIA, 1996). LEAFLET (1996) reportou tendência para melhora na conversão alimentar proporcionada pela gordura dietética em dietas contendo farelo de soja, e não uréia como fonte de nitrogênio, efeito semelhante ao 
reportado acima para monensina. CLARY et al. (1993), por sua vez, demonstraram que a monensina não apresentou benefício em melhorar a conversão alimentar quando lipídio estava presente na dieta.

Baseado nestas observações, o presente experimento objetivou estudar as interações entre monensina, óleo de soja e fonte de nitrogênio sobre o consumo de matéria seca, ganho de peso e eficiência alimentar de bovinos confinados recebendo dietas com $90 \%$ de concentrado.

\section{Material e métodos}

Quarenta novilhos Aberdeen Angus com $300 \mathrm{~kg}$ de peso corporal médio em jejum e idade de oito meses, adquiridos de uma mesma propriedade, foram usados neste estudo. Os novilhos foram confinados em baias individuais com piso ripado $(2,5 \times 3,5 \mathrm{~m})$, em área totalmente coberta e equipadas com comedouros de concreto e bebedouros automáticos, pretencentes às instalações de Gado de Corte da Universidade de Cornell, Ithaca, NY, Estados Unidos.
O experimento ocorreu nos meses de maio a agosto de 1996, onde a temperatura média das máximas era de $20^{\circ} \mathrm{C}$ e a média das mínimas de $12^{\circ} \mathrm{C}$. Três semanas antes do período experimental, os novilhos foram adaptados ao aumento gradativo do nível de concentrado. Todas as dietas experimentais continham $90 \%$ de concentrado (Tabela 1), constituídas basicamente de milho quebrado e formuladas para atender aos requerimentos nutricionais, segundo o NATIONAL RESEARCH COUNCIL - NRC (1996). Monensina foi adicionada como premix comercial para atingir a concentração desejada na dieta.

Os quarenta novilhos foram alocados aleatoriamente aos tratamentos. $\mathrm{O}$ experimento consistiu de um fatorial 2 × 2 × 2 (dois níveis de monensina - 0 e $22 \mathrm{mg} / \mathrm{kg}$ de matéria seca; dois níveis de óleo de soja - 0 e 4\%; e duas fontes de nitrogênio - farelo de soja e uréia). A alimentação era fornecida uma vez por dia e as sobras e fezes removidas sempre que acumulavam. O experimento teve duração de 112 dias, com pesagem dos animais a cada 28 dias, pela manhâ, antes da alimentação. Era descontado $4 \%$ do peso

Tabela 1 - Composição percentual e nutricional das dietas utilizadas(\%MS)

Table 1 - Percentage and nutritional composition of the used diets (\%DM)

\begin{tabular}{|c|c|c|c|c|}
\hline & \multicolumn{4}{|c|}{$\begin{array}{c}\text { Tratamentos } \\
\text { Treatment }\end{array}$} \\
\hline & $\begin{array}{c}0 \% \text { de óleo } \\
\text { (0\% oil) } \\
+ \\
\text { Uréia } \\
\text { Urea }\end{array}$ & $\begin{array}{c}0 \% \text { de óleo } \\
\quad(0 \% \text { oil }) \\
+ \\
\text { Farelo de soja } \\
\text { Soybean meal }\end{array}$ & $\begin{array}{c}\text { 4\% de óleo } \\
\text { (4\% oil) } \\
+ \\
\text { Uréia } \\
\text { Urea }\end{array}$ & $\begin{array}{c}4 \% \text { de óleo } \\
\quad \begin{array}{c}\text { (4\% oil }) \\
+ \\
\text { Farelo de soja } \\
\text { Soybean meal }\end{array}\end{array}$ \\
\hline $\begin{array}{l}\text { Silagem de milho } \\
\text { Corn silage }\end{array}$ & 15,00 & 15,00 & 15,00 & 15,00 \\
\hline $\begin{array}{l}\text { Farelo de soja } \\
\text { Soybean meal }\end{array}$ & 4,70 & 9,00 & 5,90 & 9,70 \\
\hline $\begin{array}{l}\text { Uréia } \\
\text { Urea }\end{array}$ & 0,80 & 0,00 & 0,80 & 0,00 \\
\hline $\begin{array}{l}\text { Óleo de soja } \\
\text { Soybean oil }\end{array}$ & 0,00 & 0,00 & 4,00 & 4,00 \\
\hline $\begin{array}{l}\text { Calcário } \\
\text { Limestone }\end{array}$ & 2,25 & 2,25 & 2,25 & 2,25 \\
\hline $\begin{array}{l}\text { Fosfato bicálcico } \\
\text { Dicalcium phosphate }\end{array}$ & 0,75 & 0,75 & 0,75 & 0,75 \\
\hline $\begin{array}{l}\text { Premix mineral } \\
\text { Mineral premix }\end{array}$ & 0,15 & 0,15 & 0,15 & 0,15 \\
\hline $\begin{array}{l}\text { Proteína bruta } \\
\text { Crude protein }\end{array}$ & 13,50 & 13,50 & 13,50 & 13,50 \\
\hline $\begin{array}{l}\text { Extrato etéreo } \\
\text { Ether extract }\end{array}$ & 3,00 & 3,00 & 7,00 & 7,00 \\
\hline $\begin{array}{l}\text { FDN } \\
N D F\end{array}$ & 14,00 & 14,00 & 14,00 & 14,00 \\
\hline
\end{tabular}


corporal para obter o peso corporal em jejum.

$\mathrm{Na}$ análise estatística, cada animal constituiu uma unidade experimental. Os dados foram analisados em delineamento inteiramente casualisado, usando o procedimento GLM do Minitab (RYAN e JOINER, 1994). O modelo incluiu os efeitos principais da monensina, óleo de soja, fonte de nitrogênio e suas interações. Os efeitos de tratamentos foram comparados por contrastes ortogonais completos, ao nível de 5\% de probabilidade. Quando as interações eram significativas, as médias dos tratamentos foram comparadas usando o teste de Tukey a 5\% (STEEL e TORRIE, 1960).

\section{Resultados e Discussão}

A Tabela 2 apresenta os resultados do efeito da monensina, óleo de soja e fonte de nitrogênio sobre o desempenho de novilhos Aberdeen Angus sob dietas com $90 \%$ de concentrado e $13,5 \%$ de proteína bruta. As tabelas seguintes foram apresentadas para demons- trar os efeitos significativos observados na Tabela 2.

A monensina diminuiu o consumo de matéria seca das dietas contendo farelo de soja $(\mathrm{P}<0,05)$ (Tabela $3)$, interação esta também observada por LANA et al. (1997). A redução no consumo de matéria seca causada pela monensina tem sido explicada pela redução nas perdas por fermentação e aumento na disponibilidade energética (GOODRICH et al., 1984). Entretanto, este efeito não explica a razão pela qual a monensina não reduziu o consumo de matéria seca quando a uréia foi utilizada como fonte de nitrogênio.

O nível 2 do NRC (1996) de gado de corte mostra que a degradação ruminal da matéria seca é uma propriedade do alimento, e possíveis interações entre degradação de carboidrato e proteína são ignoradas (RUSSELL et al., 1992). A observada interação entre monensina e fonte de nitrogênio sobre o consumo de matéria seca pode estar relacionada à desaminação ruminal de amino ácidos. Monensina tem, em alguns casos, diminuído a desaminação de amino ácidos e, conseqüentemente, a concentração

Tabela 2 - Efeitos da monensina, óleo de soja e fonte de nitrogênio ( $U$ =uréia e FS=farelo de soja) no consumo de matéria seca, peso corporal, ganho de peso médio diário e eficiência alimentar (ganho/consumo de MS) de novilhos alimentados com dietas contendo $90 \%$ de concentrado e $13,5 \%$ de PB

Table 2 - Effects of monensin, soybean oil and nitrogen source (U=urea e FS=soybean meal) on dry matter intake, body weight, average daily gain and feed efficiency (gain/DMI) of steers fed $90 \%$ concentrate and $13.5 \%$ CP diets

\begin{tabular}{|c|c|c|c|c|c|c|c|c|c|c|}
\hline \multirow{3}{*}{$\begin{array}{l}\text { Dias } \\
\text { Days }\end{array}$} & \multicolumn{4}{|c|}{$\begin{array}{c}0 \text { ppm de monensina } \\
\text { O ppm monensin }\end{array}$} & \multicolumn{4}{|c|}{$\begin{array}{l}22 \mathrm{ppm} \text { de monensina } \\
22 \text { ppm monensin }\end{array}$} & \multirow{3}{*}{$\begin{array}{l}\text { EPM } \\
S E M\end{array}$} & \multirow{3}{*}{$\begin{array}{l}\text { Contraste } \\
\text { significativo }^{\mathrm{a}} \\
(\mathrm{P}<0,05)\end{array}$} \\
\hline & \multicolumn{2}{|c|}{$\begin{array}{c}0 \% \text { de óleo } \\
0 \% \text { oil } \\
\end{array}$} & \multicolumn{2}{|c|}{$\begin{array}{c}4 \% \text { de óleo } \\
4 \% \text { oil }\end{array}$} & \multicolumn{2}{|c|}{$\begin{array}{c}0 \% \text { de óleo } \\
0 \% \text { oil } \\
\end{array}$} & \multicolumn{2}{|c|}{$\begin{array}{c}4 \% \text { de óleo } \\
4 \% \text { oil }\end{array}$} & & \\
\hline & $\mathrm{U}$ & FS & $\mathrm{U}$ & $\mathrm{FS}$ & $\mathrm{U}$ & FS & $\mathrm{U}$ & FS & & \\
\hline \multicolumn{11}{|c|}{ Consumo de matéria seca (Dry matter intake), kg/dia } \\
\hline d $1-56$ & 8,45 & 8,73 & 8,50 & 8,92 & 7,88 & 8,21 & 8,39 & 7,93 & 0,341 & 1 \\
\hline d 57-112 & 9,37 & 9,99 & 8,45 & 9,58 & 8,96 & 8,46 & 9,18 & 8,09 & 0,375 & 1,5 \\
\hline d $1-112$ & 8,91 & 9,45 & 8,48 & 9,25 & 8,42 & 8,27 & 8,78 & 7,98 & 0,287 & 1,5 \\
\hline \multicolumn{11}{|c|}{ Peso corporal (Body weight), $\mathrm{kg}$} \\
\hline d 1 & 302 & 295 & 302 & 300 & 287 & 292 & 303 & 305 & 5,92 & 2 \\
\hline d 56 & 397 & 406 & 398 & 395 & 381 & 384 & 395 & 387 & 6,78 & 1 \\
\hline d 112 & 480 & 485 & 462 & 471 & 457 & 461 & 470 & 444 & 10,70 & 1 \\
\hline \multicolumn{11}{|c|}{ Ganho de peso médio diário (average daily gain), kg/dia } \\
\hline d 1-56 & 1,69 & 1,92 & 1,71 & 1,70 & 1,76 & 1,70 & 1,64 & 1,46 & 0,103 & - \\
\hline d 57-112 & 1,49 & 1,41 & 1,13 & 1,37 & 1,42 & 1,30 & 1,35 & 1,02 & 0,105 & 2,5 \\
\hline d 1-112 & 1,59 & 1,69 & 1,42 & 1,53 & 1,56 & 1,50 & 1,50 & 1,24 & 0,080 & 2,5 \\
\hline \multicolumn{11}{|c|}{ Eficiência alimentar (ganho/consumo de MS) (Feed efficiency, gain/DM intake) } \\
\hline d $1-56$ & 0,193 & 0,228 & 0,197 & 0,189 & 0,208 & 0,208 & 0,195 & 0,180 & 0,009 & 2,6 \\
\hline d $57-112$ & 0,159 & 0,130 & 0,132 & 0,135 & 0,152 & 0,151 & 0,143 & 0,113 & 0,008 & $2,3,7$ \\
\hline d $1-112$ & 0,174 & 0,162 & 0,160 & 0,157 & 0,176 & 0,174 & 0,164 & 0,137 & 0,006 & $2,3,7$ \\
\hline
\end{tabular}

$\overline{\mathrm{a}} 1=$ Efeito de monensina (0 vs $22 \mathrm{mg} / \mathrm{kg}$ de MS); 2 = Efeito de óleo de soja (0 vs $4 \%$ da MS); 3 = efeito de fonte de nitrogênio (farelo de soja vs uréia); 4 = interação entre monensina e óleo de soja $(1 \times 2) ; 5=$ interação entre monensina e fonte de nitrogênio $(1 \times 3) ; 6=$ interação entre óleo de soja e fonte de nitrogênio $(2 \times 3)$; e 7 = interação entre monensina, óleo de soja e fonte de nitrogênio (1 $\times 2 \times 3)$.

a $1=$ Effect of monensin ( 0 vs $22 \mathrm{mg} / \mathrm{kg}$ of DM); 2 = effect of soybean oil ( $0 \mathrm{vs} 4 \%$ of DM); 3 = effect of nitrogen source (soybean meal vs urea); $4=$ interaction monensin vs soybean oil ( $1 \times 2) ; 5=$ interaction monensin vs nitrogen source $(1 \times 3) ; 6=$ interaction soybean oil vs nitrogen source $(2 \times 3)$; and $7=$ interaction monensin vs soybean oil vs nitrogen source $(1 \times 2 \times 3)$. 
Tabela 3 - Efeitos da monensina e fonte de nitrogênio ( $\mathrm{U}=$ uréia e $\mathrm{FS}=$ farelo de soja) no consumo de matéria seca ( $\mathrm{kg} / \mathrm{dia})$ de novilhos alimentados com dietas contendo $90 \%$ de concentrado e $13,5 \%$ de PB

Table 3 - Effects of monensin and nitrogen source ( $U=$ urea e FS=soybean meal) on dry matter intake ( $\mathrm{kg} /$ day) of steers fed $90 \%$ concentrate and $13.5 \% \mathrm{CP}$ diets

\begin{tabular}{|c|c|c|c|c|c|c|c|c|}
\hline \multirow[b]{3}{*}{$\begin{array}{l}\text { Dias } \\
\text { Days }\end{array}$} & \multirow{2}{*}{\multicolumn{2}{|c|}{$\begin{array}{c}\text { Monensina } \\
\text { Monensin }\end{array}$}} & \multicolumn{6}{|c|}{ Monensina (monensin) } \\
\hline & & & \multirow[b]{2}{*}{$\begin{array}{l}\text { EPM- } \\
S E M\end{array}$} & \multicolumn{2}{|c|}{$0 \mathrm{ppm}$} & \multicolumn{2}{|c|}{$22 \mathrm{ppm}$} & \multirow[b]{2}{*}{$\begin{array}{l}\text { EPM } \\
S E M\end{array}$} \\
\hline & $0 \mathrm{ppm}$ & $22 \mathrm{ppm}$ & & U & FS & U & FS & \\
\hline d 1-56 & $8,65^{\mathrm{a}}$ & $8,10^{\mathrm{b}}$ & 0,171 & 8,47 & 8,83 & 8,13 & 8,07 & 0,241 \\
\hline d 57-112 & $9,35^{\mathrm{a}}$ & $8,67^{\mathrm{b}}$ & 0,187 & $8,91^{\mathrm{ab}}$ & $9,78^{a}$ & $9,07^{\mathrm{ab}}$ & $8,27^{b}$ & 0,265 \\
\hline d $1-112$ & $9,02^{\mathrm{a}}$ & $8,36^{b}$ & 0,143 & $8,69^{b}$ & $9,35^{\mathrm{a}}$ & $8,60^{\mathrm{b}}$ & $8,12^{b}$ & 0,203 \\
\hline
\end{tabular}

Médias, na linha, para cada fonte de variação, seguidas por letras diferentes são diferentes $(P<0,05)$ pelo teste Tukey.

Means, within a row, for each variation source, followed by different letters are different $(P<.05)$ by Tukey test.

ruminal de amônia (GOODRICH et al., 1984; YANG e RUSSELL, 1993; LANA e RUSSELL, 1997). A redução do nível de amônia pode causar inibição da fermentação ruminal e, então, reduzir o consumo de matéria seca e o ganho de peso.

Lipídios insaturados têm efeito negativo sobre o crescimento microbiano ruminal, inibindo principalmente as bactérias gram-positivas. Embora as bactérias metanogênicas sejam altamente sensíveis aos lipídios e a redução no consumo de matéria seca por inibidores microbianos tem sido explicada pela redução nas perdas gasosas (GOODRICH et al., 1984), é surpreendente que a adição de $4 \%$ de óleo de soja na ração não tenha afetado o consumo.

A monensina diminuiu o peso corporal em jejum aos 56 e 112 dias $(\mathrm{P}<0,05)($ Tabela 4$)$, devido em parte à redução no consumo de matéria seca (Tabela 3). O óleo diminuiu o ganho de peso nos períodos de 57-112 dias e 1-112 dias (Tabela 5), enquanto a monensina diminuiu o ganho de peso nestes períodos nas dietas contendo farelo de soja como fonte de nitrogênio $(\mathrm{P}<0,05)$.
A redução do ganho de peso pelo óleo de soja pode estar relacionada à inibição da síntese de proteína microbiana ruminal, reduzindo o suprimento de proteína no intestino delgado. A diminuição do ganho de peso pela monensina, assim como do peso corporal em jejum, pode ser em parte atribuída à redução no consumo de matéria seca. A Figura 1, plotada com os dados deste experimento, corrobora esta afirmativa, na qual se observa que o aumento no consumo de matéria seca explica $57 \%$ da variação no aumento do ganho de peso.

O óleo piorou a eficiência alimentar em todos os períodos e o farelo de soja, comparado com a uréia, nos períodos de 57-112 dias e 1-112 dias $(\mathrm{P}<0,05)$ (Tabela 6). Isto ocorreu devido ao efeito mais acentuado na redução do ganho de peso em relação à redução no consumo de matéria seca. Portanto, para maximizar o ganho de peso, deve haver estímulo ao consumo de matéria seca.

Houve efeito de interação entre a monensina, óleo e fonte de nitrogênio sobre a eficiência alimentar

Tabela 4 - Efeitos da monensina e óleo de soja no peso corporal em jejum $(\mathrm{kg})$ de novilhos alimentados com dietas contendo $90 \%$ de concentrado e $13,5 \%$ de PB

Table 4 - Effects of monensin and soybean oil on the shrunk body weight $(\mathrm{kg})$ of steers fed $90 \%$ concentrate and $13.5 \%$ CP diets

\begin{tabular}{|c|c|c|c|c|c|}
\hline \multirow{2}{*}{$\begin{array}{l}\text { Dia } \\
\text { Day }\end{array}$} & \multicolumn{2}{|c|}{$\begin{array}{l}\text { Monensina } \\
\text { Monensin }\end{array}$} & \multicolumn{2}{|c|}{$\begin{array}{l}\text { Óleo de soja } \\
\text { Soybean oil }\end{array}$} & \multirow{2}{*}{$\begin{array}{l}\text { EPM } \\
S E M\end{array}$} \\
\hline & $0 \mathrm{ppm}$ & $22 \mathrm{ppm}$ & $0 \%$ & $4 \%$ & \\
\hline d 1 & 300 & 297 & $294^{a}$ & $302^{b}$ & 2,96 \\
\hline d 56 & $399^{a}$ & $387^{\mathrm{b}}$ & 392 & 394 & 3,39 \\
\hline $\mathrm{d} 112$ & $474^{\mathrm{a}}$ & $458^{\mathrm{b}}$ & 471 & 462 & 5,35 \\
\hline
\end{tabular}

Médias, na linha, para cada fonte de variação, seguidas por letras diferentes são diferentes $(P<0,05)$ pelo teste Tukey.

Means, within a row, for each variation source, followed by different letters are different $(P<.05)$ by Tukey test. 
Rev. bras. zootec.

Tabela 5 - Efeitos do óleo de soja, monensina e fonte de nitrogênio (U=uréia e FS=farelo de soja) no ganho de peso diário ( $\mathrm{kg} / \mathrm{dia})$ de novilhos alimentados com dietas contendo $90 \%$ de concentrado e $13,5 \%$ de PB

Table 5 - Effects of soybean oil, monensin and nitrogen source (U=urea e FS=soybean meal) on the average daily gain ( $\mathrm{kg} /$ day) of steers fed $90 \%$ concentrate and $13.5 \%$ CP diets

\begin{tabular}{|c|c|c|c|c|c|c|c|c|}
\hline \multirow{3}{*}{$\begin{array}{l}\text { Dias } \\
\text { Days }\end{array}$} & \multirow{2}{*}{\multicolumn{2}{|c|}{$\begin{array}{l}\text { Óleo de soja } \\
\text { Soybean oil }\end{array}$}} & \multirow{3}{*}{$\begin{array}{l}\text { EPM } \\
S E M\end{array}$} & \multicolumn{4}{|c|}{ Monensina (monensin) } & \multirow{3}{*}{$\begin{array}{c}\text { EPM } \\
S E M\end{array}$} \\
\hline & & & & \multicolumn{2}{|c|}{$0 \mathrm{ppm}$} & \multicolumn{2}{|c|}{$22 \mathrm{ppm}$} & \\
\hline & $0 \%$ & $4 \%$ & & $\mathrm{U}$ & FS & $\mathrm{U}$ & FS & \\
\hline $\mathrm{d} 1-56$ & 1,77 & 1,63 & 0,051 & 1,70 & 1,81 & 1,70 & 1,58 & 0,073 \\
\hline d 57-112 & $1,40^{\mathrm{a}}$ & $1,22^{b}$ & 0,052 & $1,31^{\mathrm{ab}}$ & $1,39^{a}$ & $1,38^{\mathrm{ab}}$ & $1,16^{\mathrm{b}}$ & 0,074 \\
\hline d 1-112 & $1,58^{\mathrm{a}}$ & $1,42^{b}$ & 0,040 & $1,50^{\mathrm{ab}}$ & $1,61^{\mathrm{a}}$ & $1,53^{\mathrm{ab}}$ & $1,37^{\mathrm{b}}$ & 0,056 \\
\hline
\end{tabular}

Médias, na linha, para cada fonte de variação, seguidas por letras diferentes são diferentes $(P<0,05)$ pelo teste Tukey.

Means, within a row, for each variation source, followed by different letters are different $(P<.05)$ by Tukey test.

( $\mathrm{P}<0,05)$, no períodos de 57-112 dias e 1-112 dias. No período de 1-112 dias, a monensina melhorou em $7,4 \%$ a eficiência alimentar de dietas contendo farelo de soja, na ausência do óleo de soja, não alterou a eficiência de dietas contendo uréia, e piorou em 13\% a eficiência alimentar de dietas contendo farelo de soja, quando o óleo de soja foi adicionado à ração (Tabela 7).

O efeito da monensina sobre a melhora da eficiência alimentar em dietas contendo farelo de soja e não uréia como fonte de nitrogênio foi também observado por HANSON e KLOPFENSTEIN (1979) e

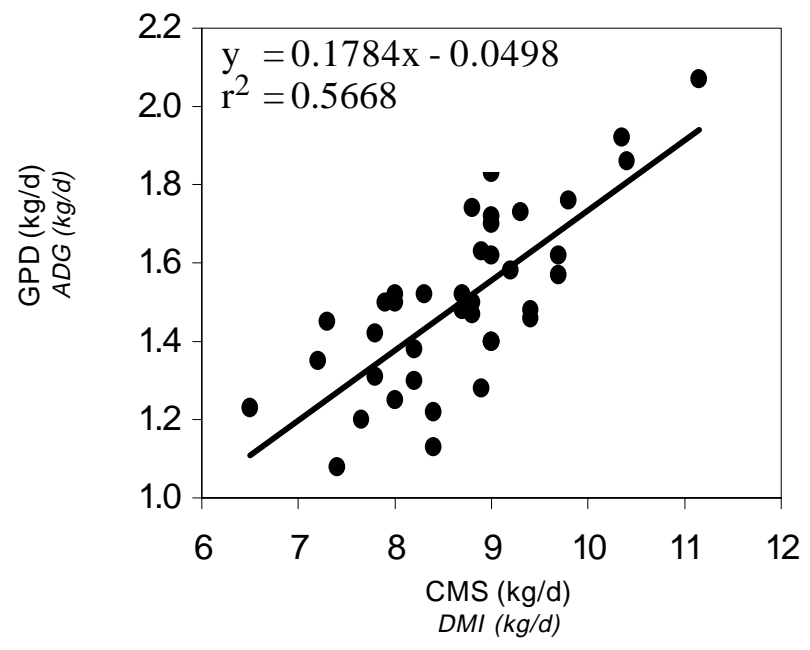

Figura 1 - Efeito do consumo de matéria seca (CMS) sobre o ganho de peso diário (GDP) de novilhos Angus alimentados com dietas contendo $90 \%$ de concentrado e $13,5 \%$ de PB.

Figure 1 - Effect of dry matter intake (DMI) on the average daily gain (ADG) of Angus steers fed $90 \%$ concentrate and $13.5 \%$ CP diets.
LANA et al. (1997). Esta interação é provavelmente devida à redução na fermentação ruminal de proteína, com conseqüente aumento no suprimento de proteína no intestino delgado e redução no custo energético para excreção urinária de uréia.

De acordo com dados publicados por CLARY et al. (1993), a monensina não apresenta benefício sobre a eficiência alimentar quando a dieta é suplementada com lipídios. Este efeito pode ser explicado pelos resultados apresentados por LEAFLET (1996), o qual observou tendência para aumento na eficiência alimentar pela adição de lipídios em dietas contendo farelo de soja e não uréia, efeito semelhante ao observado com monensina. Entretanto, neste experimento, a fonte de lipídio insaturado piorou a eficiência alimentar, principalmente quando associado à monensina, em dieta contendo farelo de soja. Este resultado foi devido provavelmente ao efeito aditivo da monensina e óleo na redução do consumo de matéria seca.

O ionóforo monensina tem decrescido seu efeito na melhora da conversão alimentar de bovinos em confinamento, desde a sua aprovação para uso na alimentação de gado de corte nos Estados Unidos, em meados da década de 70 (RAUN, 1992). Esta observação foi baseada em sumários de resultados de pesquisa analisadas e publicadas nas décadas de 70 , 80 e início de 90 . Com o passar do tempo, desde a década de 70, tem sido utilizado cada vez mais lipídios nas rações de animais em confinamentos, além de fontes de nitrogênio não protéico e elevados níveis de concentrado, visando, assim, a redução do custo com a alimentação e melhora na eficiência alimentar. Todos estes fatores contribuíram, conjuntamente, 
Tabela 6 - Efeitos do óleo de soja e fonte de nitrogênio ( $U=$ uréia e FS=farelo de soja) na eficiência alimentar (ganho/consumo de MS) de novilhos alimentados com dietas contendo $90 \%$ de concentrado e $13,5 \%$ de PB

Table 6 - Effects of soybean oil and nitrogen source (U=urea e FS=soybean meal) on the feed efficiency (gain/DMI) of steers fed 90\% concentrate and $13.5 \% \mathrm{CP}$ diets

\begin{tabular}{|c|c|c|c|c|c|}
\hline \multirow{2}{*}{$\begin{array}{l}\text { Dias } \\
\text { Days }\end{array}$} & \multicolumn{2}{|c|}{$\begin{array}{l}\text { Óleo de soja } \\
\text { Soybean oil }\end{array}$} & \multicolumn{2}{|c|}{$\begin{array}{c}\text { Fonte de nitrogênio } \\
\text { Nitrogen source }\end{array}$} & \multirow{2}{*}{$\begin{array}{l}\text { EPM } \\
S E M\end{array}$} \\
\hline & $\%$ & $4 \%$ & $\mathrm{U}$ & FS & \\
\hline d 1-56 & $0,209^{\mathrm{a}}$ & $0,190^{\mathrm{b}}$ & 0,198 & 0,201 & 0,004 \\
\hline d 57-112 & $0,148^{\mathrm{a}}$ & $0,131^{\mathrm{b}}$ & $0,146^{\mathrm{a}}$ & $0,132^{\mathrm{b}}$ & 0,004 \\
\hline d 1-112 & $0,171^{\mathrm{a}}$ & $0,154^{\mathrm{b}}$ & $0,168^{a}$ & $0,157^{b}$ & 0,003 \\
\hline
\end{tabular}

Médias, na linha, para cada fonte de variação, seguidas por letras diferentes são diferentes $(\mathrm{P}<0,05)$ pelo teste de Tukey. Means, within a row, for each variation source, followed by different letters are different $(P<.05)$ by Tukey test.

Tabela 7 - Efeitos da monensina, óleo de soja e fonte de nitrogênio ( $U=$ uréia e FS=farelo de soja) na eficiência alimentar (ganho/consumo de MS) de novilhos alimentados com dietas contendo $90 \%$ de concentrado e $13,5 \%$ de PB

Table 7 - Effects of monensin, soybean oil and nitrogen source (U=urea e FS=soybean meal) on the feed efficiency (gain/DMI) of steers fed $90 \%$ concentrate and $13.5 \% \mathrm{CP}$ diets

\begin{tabular}{|c|c|c|c|c|c|c|c|c|c|}
\hline \multirow{3}{*}{$\begin{array}{l}\text { Dias } \\
\text { Days }\end{array}$} & \multicolumn{4}{|c|}{$\begin{array}{c}0 \text { ppm de monensina } \\
\text { O ppm monensin }\end{array}$} & \multicolumn{4}{|c|}{$\begin{array}{l}22 \text { ppm de monensina } \\
22 \text { ppm monensin }\end{array}$} & \multirow{3}{*}{$\begin{array}{l}\text { EPM } \\
\text { SEM }\end{array}$} \\
\hline & \multicolumn{2}{|c|}{$\begin{array}{c}0 \% \text { de óleo } \\
\text { o\% oil }\end{array}$} & \multicolumn{2}{|c|}{$\begin{array}{c}4 \% \text { de óleo } \\
4 \% \text { oil }\end{array}$} & \multicolumn{2}{|c|}{$\begin{array}{c}0 \% \text { de óleo } \\
\text { o\% oil }\end{array}$} & \multicolumn{2}{|c|}{$\begin{array}{c}4 \% \text { de óleo } \\
4 \% \text { oil }\end{array}$} & \\
\hline & $\mathrm{U}$ & FS & $\mathrm{U}$ & FS & $\mathrm{U}$ & FS & $\mathrm{U}$ & FS & \\
\hline $\mathrm{d} 1-56$ & 0,193 & 0,228 & 0,197 & 0,189 & 0,208 & 0,208 & 0,195 & 0,180 & 0,009 \\
\hline d 57-112 & 0,159 & 0,130 & 0,132 & 0,135 & 0,152 & 0,151 & 0,143 & 0,113 & 0,008 \\
\hline d 1-112 & 0,174 & 0,162 & 0,160 & 0,157 & 0,176 & 0,174 & 0,164 & 0,137 & 0,006 \\
\hline
\end{tabular}

para reduzir o benefício da monensina na melhora da eficiência alimentar de bovinos em confinamento, conforme pode ser observado nos resultados desta pesquisa, pelo uso de fonte de nitrogênio não protéico e pela presença de lipídios.

\section{Conclusões}

Monensina é mais eficiente em melhorar a conversão alimentar de animais em confinamento quando á dieta é suplementada com fontes de proteína verdadeira, como o farelo de soja, e sem inclusão de lipídios. Uma vez que os lipídios têm modo de ação sobre os microrganismos ruminais semelhantes aos ionóforos, os mesmos apresentam efeito aditivo, podendo prejudicar a fermentação ruminal e piorar a conversão alimentar, quando usados conjuntamente.

\section{Referências Bibliográficas}

CHALUPA, W., RICKABAUGH, B., KRONFELD, D.S. et al. 1984. Rumen fermentation in vitro as influenced by long chain fatty acids. J. Dairy Sci., 67:1439-1444.

CLARY, E.M., BRANDT, R.T., HARMON, D.L. et al. 1993. Supplemental fat and ionophores in finishing diets: feedlot performance and ruminal digesta kinetics in steers. J. Anim. Sci., 71:3115-3123

DINIUS, D.A., SIMPSON, M.E., MARSH, P.B. 1976. Effect of monensin fed with forage on digestion and the ruminal ecosystem of steers. J. Anim. Sci., 42:229-234.

GOODRICH, R.D., GARRETT, J.E., GAST, D.R. et al. 1984 Influence of monensin on the performance of cattle. J. Anim. Sci. 58:1484-1498.

HANSON, T.L., KLOPFENSTEIN, T. 1979. Monensin, protein source and protein levels for growing steers. J. Anim. Sci., 48:474-479.

KRAUSE, D.O., RUSSELL, J.B. 1996. An rRNA approach for assessing the role of obligate amino acid-fermenting bacteria in ruminal amino acid deamination. Appl. Environ. Microbiol., 62(3):815-821. 
Rev. bras. zootec.

LANA, R.P., FOX, D.G., RUSSELL, J.B. et al. 1997. Influence of monensin on Holstein steers fed high-concentrate diets containing soybean meal or urea. J. Anim. Sci., 75:2571-2579.

LANA, R.P., RUSSELL, J.B. 1997. Effect of forage quality and monensin on the ruminal fermentation of fistulated cows fed continuously at a constant intake. J. Anim. Sci., 75:224-229.

LEAFLET, A.S. Responses to adding fat to corn-based diets supplemented with urea and soybean meal for finishing steers. In: 1996 BEEF RESEARCH REPORT, 1996, Iowa, USA. Proceedings... Iowa State University, 1996. p.71-74.

NATIONAL RESEARCH COUNCIL - NRC. 1996. Nutrient requirements of beef cattle. 7.ed. Washington, DC: National Academy Press. 242p.

RAUN, A.P. Rumensin "then and now". In: RUMENSIN "IN THE 1990's”, 1990, Dallas, TX. Proceedings... Indianápolis: Elanco Animal Health, 1992, p. A1-A20.

RICHARDSON, L.F., RAUN, A.P., POTTER, E.L. et al. 1976. Effect of monensin on ruminal fermentation in vitro and in vivo. J. Anim. Sci., 43:657-664.

RUSSELL, J.B., O'CONNOR, J.D., FOX, D.G. et al. 1992. A net carbohydrate and protein system for evaluating cattle diets. I. Ruminal fermentation. J. Anim. Sci., 70:3551-3561.

RUSSELL, J.B., STROBEL, H.J. 1989. The effect of ionophores on rumen fermentation. Appl. Environ. Microbiol., 55:1-6.
RYAN, B.F., JOINER, B.L. 1994. Minitab handbook. 3.ed. Belmont, CA Duxbury Press. 448p.

STEEL, R.G.D., TORRIE, J.H. 1960. Principles and procedures of statistics. New York: McGraw-Hill. 48p.

THORNTON, J.H., OWENS, F.N. 1981. Monensin supplementation and in vivo methane production by steers. J. Anim. Sci., 51:628-634.

WEDEGAERTNER, T.C., JOHNSON, D.E. 1983. Monensin effects on digestibility, methanogenesis, and heat increment of a cracked corn-silage diet fed to steers. J. Anim. Sci., 57:168-177.

YANG, C.M.J., RUSSELL, J.B. 1993. The effect of monensin supplementation on ruminal ammonia accumulation in vivo and the numbers of amino acid-fermenting bacteria. J. Anim. Sci., 71:3470-3476.

ZINN, R.A., PLASCENCIA, A. 1996. Effects of forage level on the comparative feeding value of supplemental fat in growingfinishing diets for feedlot cattle. J. Anim. Sci., 74:1194.

Recebido em: 08/02/00

Aceito em: 21/09/00 